\title{
Existence of Traveling Wave Solutions for a Nonlocal Bistable Equation: An Abstract Approach
}

\author{
By
}

\author{
Hiroki Yagisita*
}

\begin{abstract}
We consider traveling fronts to the nonlocal bistable equation

$$
u_{t}=\mu * u-u+f(u),
$$

where $\mu$ is a Borel-measure on $\mathbb{R}$ with $\mu(\mathbb{R})=1$ and $f$ satisfies $f(0)=f(1)=0, f<0$ in $(0, \alpha)$ and $f>0$ in $(\alpha, 1)$ for some constant $\alpha \in(0,1)$. We do not assume that $\mu$ is absolutely continuous with respect to the Lebesgue measure. We show that there are a constant $c$ and a monotone function $\phi$ with $\phi(-\infty)=0$ and $\phi(+\infty)=1$ such that $u(t, x):=\phi(x+c t)$ is a solution to the equation, provided $f^{\prime}(\alpha)>0$. In order to prove this result, we would develop a recursive method for abstract monotone dynamical systems and apply it to the equation.
\end{abstract}

\section{$\S 1 . \quad$ Introduction}

This paper is a sequel to [18], where the author has considered a nonlocal analogue of monostable reaction-diffusion equations. In this paper, we would consider the following nonlocal analogue of bistable reaction-diffusion equations:

$$
u_{t}=\mu * u-u+f(u)
$$

Communicated by H. Okamoto. Received November 1, 2008. Revised February 28, 2009. 2000 Mathematics Subject Classification(s): 35K57, 35K65, 35K90, 45J05.

Key words: nonlocal phase transition, Ising model, convolution model, integro-differential equation, discrete bistable equation, nonlocal evolution equation.

*Department of Mathematics, Faculty of Science, Kyoto Sangyo University Motoyama, Kamigamo, Kita-Ku, Kyoto-City, 603-8555, Japan.

e-mail: hrk0ygst@cc.kyoto-su.ac.jp

(c) 2009 Research Institute for Mathematical Sciences, Kyoto University. All rights reserved. 
Here, $\mu$ is a Borel-measure on $\mathbb{R}$ with $\mu(\mathbb{R})=1$ and the convolution is defined by

$$
(\mu * u)(x)=\int_{y \in \mathbb{R}} u(x-y) d \mu(y)
$$

for a bounded and Borel-measurable function $u$ on $\mathbb{R}$. The nonlinearity $f$ is a Lipschitz continuous function on $\mathbb{R}$ and satisfies $f(0)=f(\alpha)=f(1)=0$, $f<0$ in $(0, \alpha)$ and $f>0$ in $(\alpha, 1)$ for some constant $\alpha \in(0,1)$. Then, $G(u):=$ $\mu * u-u+f(u)$ is a map from the Banach space $L^{\infty}(\mathbb{R})$ into $L^{\infty}(\mathbb{R})$ and it is Lipschitz continuous. (We note that $u(x-y)$ is a Borel-measurable function on $\mathbb{R}^{2}$, and $\|u\|_{L^{\infty}(\mathbb{R})}=0$ implies $\|\mu * u\|_{L^{1}(\mathbb{R})} \leq \int_{y \in \mathbb{R}}\left(\int_{x \in \mathbb{R}}|u(x-y)| d x\right) d \mu(y)=0$. $)$ So, because the standard theory of ordinary differential equations works, we have well-posedness of the equation (1.1) and it generates a flow in $L^{\infty}(\mathbb{R})$.

In this paper, we would show that there exists a traveling wave solution. The main result is the following:

Theorem 1. Suppose the bistable nonlinearity $f \in C^{1}(\mathbb{R})$ satisfies

$$
f^{\prime}(\alpha)>0
$$

where $\alpha$ is the unique zero of $f$ in $(0,1)$. Then, there exist a constant $c$ and a monotone function $\phi$ on $\mathbb{R}$ with $\phi(-\infty)=0$ and $\phi(+\infty)=1$ such that $u(t, x):=\phi(x+c t)$ is a solution to (1.1).

In this result, we do not assume that the measure $\mu$ is absolutely continuous with respect to the Lebesgue measure. For example, Theorem 1 can be applied to not only the integro-differential equation

$$
\frac{\partial u}{\partial t}(t, x)=\int_{0}^{1} u(t, x-y) d y-u(t, x)-\lambda u(t, x)(u(t, x)-\alpha)(u(t, x)-1)
$$

but also the discrete equation

$$
\frac{\partial u}{\partial t}(t, x)=u(t, x-1)-u(t, x)-\lambda u(t, x)(u(t, x)-\alpha)(u(t, x)-1)
$$

for all positive constants $\lambda$. In order to prove Theorem 1, we would develop a recursive method for abstract monotone dynamical systems and apply it to the semiflow generated by (1.1). It might be a generalization of the method of Remark 5.2 (4) in Chen [6].

For the nonlocal bistable equation (1.1), Bates, Fife, Ren and Wang [5] obtained existence of traveling wave solutions, when the measure $\mu$ has a density function $J \in C^{1}(\mathbb{R})$ with $J(y)=J(-y)$ and other little conditions for $\mu$ and 
$f$ hold. Chen [6] showed existence of traveling wave solutions, when it has a density function $J \in C^{1}(\mathbb{R})$ and $f^{\prime}(u)<1$ and other little conditions hold. Recently, Coville [12] proved existence of traveling wave solutions, when it has a density function $J \in C(\mathbb{R})$ and other little conditions hold.

Bates, Fife, Ren and Wang [5] and Chen [6] studied uniqueness and stability of traveling wave solutions. Coville studied uniqueness and monotonicity of profiles of traveling waves in [11] and uniqueness of speeds [12]. Further, we note that the studies of $[11,12]$ are not limited when the nonlinearity is bistable but reach ignition, while our study is limited to bistable. In fact, his method of [12] is rather different from ours. See [10] on traveling wave solutions in bistable maps, [2] time-periodic nonlocal bistable equations, [1] time-periodic bistable reaction-diffusion equations, e.g., [3, 4, 7, 9, 15] discrete bistable equations, [8] nonlocal Burgers equations and $[13,14,16]$ multistable reaction-diffusion equations.

This paper is a sequel to [18], and we shall refer several known results from [18]. In Section 2, we give abstract conditions and state that there exists a traveling wave solution provided the conditions. This result might generalize the method of Remark 5.2 (4) in Chen [6]. In Section 3, we prove abstract theorems mentioned in Section 2. In Section 4, we show that the semiflow generated by (1.1) satisfies the conditions given in Section 2 when $f^{\prime}(\alpha)>0$ and $\mu(\{0\}) \neq 1$ hold to prove Theorem 1 .

\section{§2. Abstract Theorems for Monotone Semiflows}

In this section, we would state some abstract results for existence of traveling waves in monotone semiflows. The results might generalize the method of Remark 5.2 (4) in Chen [6]. In the abstract, we would treat a bistable evolution system. Put a set of functions on $\mathbb{R}$;

$$
\mathcal{M}:=\{u \mid u \text { is a monotone nondecreasing }
$$

and left continuous function on $\mathbb{R}$ with $0 \leq u \leq 1\}$.

The followings are our basic conditions for discrete dynamical systems:

Hypotheses 2. Let $Q_{0}$ be a map from $\mathcal{M}$ into $\mathcal{M}$.

(i) $Q_{0}$ is continuous in the following sense: If a sequence $\left\{u_{k}\right\}_{k \in \mathbb{N}} \subset \mathcal{M}$ converges to $u \in \mathcal{M}$ uniformly on every bounded interval, then the sequence $\left\{Q_{0}\left[u_{k}\right]\right\}_{k \in \mathbb{N}}$ converges to $Q_{0}[u]$ almost everywhere.

(ii) $Q_{0}$ is order preserving; i.e.,

$$
u_{1} \leq u_{2} \Longrightarrow Q_{0}\left[u_{1}\right] \leq Q_{0}\left[u_{2}\right]
$$


for all $u_{1}$ and $u_{2} \in \mathcal{M}$. Here, $u \leq v$ means that $u(x) \leq v(x)$ holds for all $x \in \mathbb{R}$.

(iii) $Q_{0}$ is translation invariant; i.e.,

$$
T_{x_{0}} Q_{0}=Q_{0} T_{x_{0}}
$$

for all $x_{0} \in \mathbb{R}$. Here, $T_{x_{0}}$ is the translation operator defined by $\left(T_{x_{0}}[u]\right)(\cdot):=$ $u\left(\cdot-x_{0}\right)$.

(iv) $Q_{0}$ is bistable; i.e., there exists $\alpha \in(0,1)$ with $Q_{0}[\alpha]=\alpha$ such that

$$
0<\gamma<\alpha \Longrightarrow Q_{0}[\gamma]<\gamma
$$

and

$$
\alpha<\gamma<1 \Longrightarrow \gamma<Q_{0}[\gamma]
$$

hold for all constant functions $\gamma$.

Remark. If $Q_{0}$ satisfies Hypothesis 2 (iii), then $Q_{0}$ maps constant functions to constant functions.

The following condition for discrete dynamical systems might be a little generalization of the condition in Remark 5.2 (4) of Chen [6]:

Hypothesis 3. Let $Q_{0}$ be a map from $\mathcal{M}$ into $\mathcal{M}$. If two constants $c_{-}$ and $c_{+}$and two functions $\phi_{-}$and $\phi_{+} \in \mathcal{M}$ satisfy $\left(Q_{0}\left[\phi_{-}\right]\right)\left(x-c_{-}\right) \equiv \phi_{-}(x)$, $\phi_{-}(-\infty)=0, \phi_{-}(+\infty)=\alpha,\left(Q_{0}\left[\phi_{+}\right]\right)\left(x-c_{+}\right) \equiv \phi_{+}(x), \phi_{+}(-\infty)=\alpha$ and $\phi_{+}(+\infty)=1$, then the inequality $c_{-}<c_{+}$holds.

The following states that existence of suitable sub and super-solutions implies existence of traveling wave solutions with an estimate of the speeds in the discrete dynamical systems on $\mathcal{M}$ :

Theorem 4. Let a map $Q_{0}: \mathcal{M} \rightarrow \mathcal{M}$ satisfy Hypotheses 2 and 3. Suppose a constant $\underline{c}$ and a function $\underline{\psi} \in \mathcal{M}$ with $\underline{\psi}(0)=0$ and $\underline{\psi}(+\infty) \in(\alpha, 1]$ satisfy $\psi(x) \leq\left(Q_{0}[\psi]\right)(x-\underline{c})$. Suppose a constant $\bar{c}$ and a function $\bar{\psi} \in \mathcal{M}$ with $\bar{\psi}(-\infty) \in[0, \bar{\alpha})$ and $\bar{\psi}(0)=1$ satisfy $\left(Q_{0}[\bar{\psi}]\right)(x-\bar{c}) \leq \bar{\psi}(x)$. Then, there exist $c \in[\underline{c}, \bar{c}]$ and $\phi \in \mathcal{M}$ with $\phi(-\infty)=0$ and $\phi(+\infty)=1$ such that $\left(Q_{0}[\phi]\right)(x-c) \equiv \phi(x)$ holds.

Corollary 5. Let a map $Q_{0}: \mathcal{M} \rightarrow \mathcal{M}$ satisfy Hypotheses 2 and 3 . Then, there exist $c \in \mathbb{R}$ and $\phi \in \mathcal{M}$ with $\phi(-\infty)=0$ and $\phi(+\infty)=1$ such that $\left(Q_{0}[\phi]\right)(x-c) \equiv \phi(x)$ holds. 


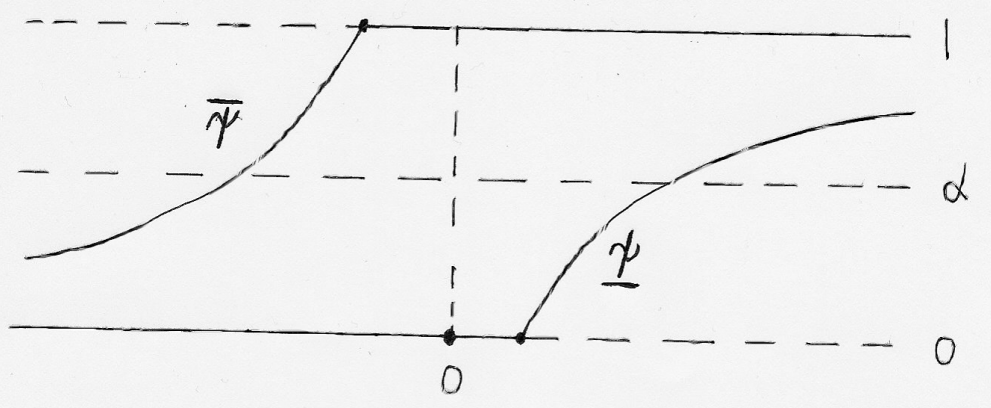

Figure 1. Typical profiles of the functions $\underline{\psi}$ and $\bar{\psi}$ in Theorems 4 and 8

We add the following conditions to Hypotheses 2 for continuous dynamical systems on $\mathcal{M}$ :

Hypotheses 6. Let $Q:=\left\{Q^{t}\right\}_{t \in[0,+\infty)}$ be a family of maps from $\mathcal{M}$ to $\mathcal{M}$.

(i) $Q$ is a semigroup; i.e., $Q^{t} \circ Q^{s}=Q^{t+s}$ for all $t$ and $s \in[0,+\infty)$.

(ii) $Q$ is continuous in the following sense: Suppose a sequence $\left\{t_{k}\right\}_{k \in \mathbb{N}} \subset$ $[0,+\infty)$ converges to 0 , and $u \in \mathcal{M}$. Then, the sequence $\left\{Q^{t_{k}}[u]\right\}_{k \in \mathbb{N}}$ converges to $u$ almost everywhere.

Instead of Hypothesis 3, we consider the following condition for continuous dynamical systems. It might also be a little generalization of the condition in Remark 5.2 (4) of Chen [6]:

Hypothesis 7. Let $Q:=\left\{Q^{t}\right\}_{t \in[0,+\infty)}$ be a family of maps from $\mathcal{M}$ to $\mathcal{M}$. If two constants $c_{-}$and $c_{+}$and two functions $\phi_{-}$and $\phi_{+} \in \mathcal{M}$ with $\phi_{-}(-\infty)=0, \phi_{-}(+\infty)=\alpha, \phi_{+}(-\infty)=\alpha$ and $\phi_{+}(+\infty)=1$ satisfy $\left(Q^{t}\left[\phi_{-}\right]\right)\left(x-c_{-} t\right) \equiv \phi_{-}(x)$ and $\left(Q^{t}\left[\phi_{+}\right]\right)\left(x-c_{+} t\right) \equiv \phi_{+}(x)$ for all $t \in[0,+\infty)$, then the inequality $c_{-}<c_{+}$holds.

Remark. In $[13,14,16]$, we could found similar hypotheses as Hypothesis 7 for existence of traveling waves to reaction-diffusion equations with triple stable equilibria. 
As we would have Theorem 4 for the discrete dynamical systems, we would have the following for the continuous dynamical systems:

Theorem 8. Let $Q^{t}$ be a map from $\mathcal{M}$ to $\mathcal{M}$ for $t \in[0,+\infty)$. Suppose the map $Q^{t}$ satisfies Hypotheses 2 for all $t \in(0,+\infty)$, and the family $Q:=$ $\left\{Q^{t}\right\}_{t \in[0,+\infty)}$ Hypotheses 6 and 7 . Then, the following holds:

Suppose a constant $\underline{c}$ and a function $\psi \in \mathcal{M}$ with $\psi(0)=0$ and $\psi(+\infty) \in$ $(\alpha, 1]$ satisfy $\underline{\psi}(x) \leq\left(Q^{t}[\underline{\psi}]\right)(x-\underline{c} t)$ for all $t \in[0,+\infty)$. Suppose a constant $\bar{c}$ and a function $\bar{\psi} \in \mathcal{M}$ with $\bar{\psi}(-\infty) \in[0, \alpha)$ and $\bar{\psi}(0)=1$ satisfy $\left(Q^{t}[\bar{\psi}]\right)(x-$ $\bar{c} t) \leq \bar{\psi}(x)$ for all $t \in[0,+\infty)$. Then, there exist $c \in[\underline{c}, \bar{c}]$ and $\phi \in \mathcal{M}$ with $\phi(-\infty)=0$ and $\phi(+\infty)=1$ such that $\left(Q^{t}[\phi]\right)(x-c t) \equiv \phi(x)$ holds for all $t \in[0,+\infty)$.

\section{§3. Proof of the Abstract Theorems}

In this section, we prove the theorems stated in Section 2. In the proof, we shall refer some known results from Sections 2 and 3 of [18].

Proof of Theorem 4.

[Step 0] In this step, we would give an intuitive explanation of our ideas. If you want to advance to exact proof at once, the step is recommended to be skipped.

Because the map $Q_{0}: \mathcal{M} \rightarrow \mathcal{M}$ is translation invariant, it is difficult to construct traveling sub and super-solutions with the same speed directly. So, we introduce a sequence of perturbed maps $Q_{n}: \mathcal{M} \rightarrow \mathcal{M}$ to break the translation invariance but to preserve the order. Then, we might construct sub and supersolutions $\underline{\psi}_{n}$ and $\bar{\psi}_{n}$ to the perturbed problem $Q_{n}[u]=u$ and also obtain a solution $\phi_{n}$ (i.e., $Q_{n}\left[\phi_{n}\right]=\phi_{n}, \phi_{n}(-\infty)=0$ and $\phi_{n}(+\infty)=1$ ) by order preserving property. In virtue of Hypothesis 3, we expect that the limit of a suitable subsequence of $\left(T_{-x_{n}}\left[\phi_{n}\right]\right)(\cdot):=\phi_{n}\left(\cdot+x_{n}\right)$ solves the original problem.

We shall explain more in detail but extremely inexactly. Let $n \in \mathbb{N}$. We put $\rho_{n}(x):=\left(1+\frac{\bar{c}-\underline{c}}{2 n}\right)\left(x-\frac{\underline{c}+\bar{c}}{2}\right)$. Then, the map $u \mapsto Q_{n}[u]:=Q_{0}\left[u \circ \rho_{n}\right]$ breaks the translation invariance but preserves the order. So, we may have a solution $\phi_{n}$ to $Q_{n}\left[\phi_{n}\right]=\phi_{n}, \phi_{n}(-\infty)=0$ and $\phi_{n}(+\infty)=1$. We take $y_{n}$ and $z_{n}$ such that $y_{n} \leq z_{n}$ and $0<\phi_{n}\left(y_{n}\right)<\alpha<\phi_{n}\left(z_{n}\right)<1$ hold.

When a constant $c$ and a sequence $x_{n}$ satisfy the equality $c=\frac{c+\bar{c}}{2}-$ $\frac{\bar{c}-\underline{c}}{2}\left(\lim _{n \rightarrow \infty} \frac{x_{n}}{n}\right)$, we see

$$
\lim _{n \rightarrow \infty}\left(\rho_{n}\left(x+x_{n}\right)-x_{n}\right)=x-c
$$


and, so,

$$
\begin{gathered}
\lim _{n \rightarrow \infty}\left(T_{-x_{n}} \circ Q_{n} \circ T_{x_{n}}\right)[u]=\lim _{n \rightarrow \infty} T_{-x_{n}}\left[Q_{0}\left[\left(T_{x_{n}}[u]\right) \circ \rho_{n}\right]\right] \\
=\lim _{n \rightarrow \infty} Q_{0}\left[T_{-x_{n}}\left[\left(T_{x_{n}}[u]\right) \circ \rho_{n}\right]=Q_{0}\left[\lim _{n \rightarrow \infty} T_{-x_{n}}\left[\left(T_{x_{n}}[u]\right) \circ \rho_{n}\right]\right]\right. \\
=Q_{0}\left[T_{c}[u]\right]=\left(Q_{0} \circ T_{c}\right)[u],
\end{gathered}
$$

where $\left(T_{x}[u]\right)(\cdot):=u(\cdot-x)$. We might take a subsequence $n(k)$ such that there exist the limits $\phi_{-}:=\lim _{k \rightarrow \infty} T_{-y_{n}}\left[\phi_{n}\right], \phi_{+}:=\lim _{k \rightarrow \infty} T_{-z_{n}}\left[\phi_{n}\right], c_{-}:=$ $\frac{\underline{c}+\bar{c}}{2}-\frac{\bar{c}-\underline{c}}{2}\left(\lim _{k \rightarrow \infty} \frac{y_{n}}{n}\right)$ and $c_{+}:=\frac{\underline{c}+\bar{c}}{2}-\frac{\bar{c}-\underline{c}}{2}\left(\lim _{k \rightarrow \infty} \frac{z_{n}}{n}\right)$.

Therefore, we could expect that the two equalities

$$
\begin{gathered}
\left(Q_{0} \circ T_{c_{-}}\right)\left[\phi_{-}\right]=\lim _{n \rightarrow \infty}\left(T_{-y_{n}} \circ Q_{n} \circ T_{y_{n}}\right)\left[\phi_{-}\right]=\lim _{k \rightarrow \infty}\left(T_{-y_{n}} \circ Q_{n}\right)\left[\phi_{n}\right] \\
=\lim _{k \rightarrow \infty} T_{-y_{n}}\left[Q_{n}\left[\phi_{n}\right]\right]=\lim _{k \rightarrow \infty} T_{-y_{n}}\left[\phi_{n}\right]=\phi_{-}
\end{gathered}
$$

and

$$
\left(Q_{0} \circ T_{c_{+}}\right)\left[\phi_{+}\right]=\phi_{+}
$$

hold. In virtue of Hypothesis 3, the pair $\left(\phi_{-}, c_{-}\right)$or $\left(\phi_{+}, c_{+}\right)$might solve the original problem, as we obtained $c_{+} \leq c_{-}$and $0<\phi_{-}(0)<\alpha<\phi_{+}(0)<1$.

[Step 1] We show the inequality:

$$
\underline{c} \leq \bar{c} .
$$

Suppose $\bar{c}<\underline{c}$. Then, there exists $N \in \mathbb{N}$ such that $\bar{\psi}\left(-\frac{\underline{c}-\bar{c}}{2} N\right)$ $<\alpha<\underline{\psi}\left(+\frac{\underline{\underline{c}-\bar{c}}}{2} N\right)$ holds. Hence, because $\left(Q_{0}{ }^{N}[\bar{\psi}]\right)(x-\bar{c} N) \leq \bar{\psi}(x)$ and $\underline{\psi}(x) \leq$ $\left(Q_{0}{ }^{N}[\overline{\psi]})(x-\underline{c} N)\right.$ hold by Hypotheses 2 (ii) and (iii), we have $\left(Q_{0}{ }^{N}[\bar{\psi}]\right)\left(-\frac{\overline{\underline{c}}+\bar{c}}{2} N\right)$ $<\alpha<\left(Q_{0}{ }^{N}[\underline{\psi}]\right)\left(-\frac{c+\bar{c}}{2} N\right)$. It is a contradiction with Hypothesis 2 (ii). Therefore, (3.1) holds.

[Step 2] We put a sequence $\left\{\rho_{n}\right\}_{n \in \mathbb{N}}$ of affine functions on $\mathbb{R}$ defined by

$$
\rho_{n}(x):=\left(1+\frac{\bar{c}-\underline{c}}{2 n}\right)\left(x-\frac{\underline{c}+\bar{c}}{2}\right) .
$$

We define two sequences $\left\{A_{n}\right\}_{n \in \mathbb{N}}$ and $\left\{Q_{n}\right\}_{n \in \mathbb{N}}$ of maps from $\mathcal{M}$ to $\mathcal{M}$ by

$$
A_{n}[u]:=u \circ \rho_{n}
$$

and

$$
Q_{n}:=Q_{0} \circ A_{n} .
$$

Then, the map $Q_{n}$ satisfies Hypothesis 2 (ii) for all $n \in \mathbb{N}$. 
[Step 3] We show the following: Suppose a sequence $\left\{u_{k}\right\}_{k \in \mathbb{N}} \subset \mathcal{M}$ converges to $u \in \mathcal{M}$ almost everywhere. Then, $\lim _{k \rightarrow \infty}\left(Q_{n}\left[u_{k}\right]\right)(x)=\left(Q_{n}[u]\right)(x)$ holds for all $n \in \mathbb{N}$ and continuous points $x \in \mathbb{R}$ of $Q_{n}[u]$.

Let $n \in \mathbb{N}$. Then, the sequence $\left\{A_{n}\left[u_{k}\right]\right\}_{k \in \mathbb{N}} \subset \mathcal{M}$ converges to $A_{n}[u] \in$ $\mathcal{M}$ almost everywhere. Hence, by Proposition 9 of [18], we have $\lim _{k \rightarrow \infty}\left(Q_{0}\right.$ $\left.\left[A_{n}\left[u_{k}\right]\right]\right)(x)=\left(Q_{0}\left[A_{n}[u]\right]\right)(x)$ for all continuous points $x \in \mathbb{R}$ of $Q_{0}\left[A_{n}[u]\right]$.

[Step 4] We take two sequences $\left\{\underline{\psi}_{n}\right\}_{n \in \mathbb{N}}$ and $\left\{\bar{\psi}_{n}\right\}_{n \in \mathbb{N}} \subset \mathcal{M}$ as

$$
\underline{\psi}_{n}(x):=\underline{\psi}\left(x-\left(n+\frac{\bar{c}-\underline{c}}{2}\right)\right)
$$

and

$$
\bar{\psi}_{n}(x):=\bar{\psi}\left(x+\left(n+\frac{\bar{c}-\underline{c}}{2}\right)\right) .
$$

Then, we show $\underline{\psi}_{n} \leq Q_{n}{ }^{k}\left[\underline{\psi}_{n}\right] \leq Q_{n}{ }^{k+1}\left[\underline{\psi}_{n}\right] \leq Q_{n}{ }^{k+1}\left[\bar{\psi}_{n}\right] \leq Q_{n}{ }^{k}\left[\bar{\psi}_{n}\right] \leq \bar{\psi}_{n}$ for all $k=0,1,2, \cdots$. By (3.1) and $(3.2),+n \leq x-\frac{\underline{c}+\bar{c}}{2}$ implies $x-\underline{c}=$ $\left(x-\frac{\underline{c}+\bar{c}}{2}\right)+\frac{\bar{c}-\underline{c}}{2} \leq \rho_{n}(x)$ and $\underline{\psi}_{n}(x-\underline{c}) \leq\left(A_{n}\left[\underline{\psi}_{n}\right]\right)(x)$. So, because $x-\frac{\underline{c}+\bar{c}}{2} \leq+n$ implies $\underline{\psi}_{n}(x-\underline{c})=\underline{\psi}\left(x-\frac{\underline{\underline{c}+\bar{c}}}{2}-n\right) \leq \underline{\psi}(0)=0$,

$$
\underline{\psi}_{n}(x-\underline{c}) \leq\left(A_{n}\left[\underline{\psi}_{n}\right]\right)(x)
$$

holds. Because $x-\frac{c+\bar{c}}{2} \leq-n$ implies $\rho_{n}(x) \leq\left(x-\frac{c+\bar{c}}{2}\right)-\frac{\bar{c}-\underline{c}}{2}=x-\bar{c}$ by $(3.1)$ and (3.2), we also see

$$
\left(A_{n}\left[\bar{\psi}_{n}\right]\right)(x) \leq \bar{\psi}_{n}(x-\bar{c}) .
$$

From (3.3), (3.4) and $\underline{\psi}_{n} \leq \bar{\psi}_{n}$, we have $\underline{\psi}_{n}(x) \leq\left(Q_{0}\left[\underline{\psi}_{n}\right]\right)(x-\underline{c}) \leq\left(Q_{n}\left[\underline{\psi}_{n}\right]\right)(x)$ $\leq\left(Q_{n}\left[\bar{\psi}_{n}\right]\right)(x) \leq\left(Q_{0}\left[\bar{\psi}_{n}\right]\right)(x-\bar{c}) \leq \bar{\psi}_{n}(x)$. As $\underline{\psi}_{n} \leq Q_{n}{ }^{k}\left[\underline{\psi}_{n}\right] \leq Q_{n}{ }^{k+1}\left[\underline{\psi}_{n}\right] \leq$ ${Q_{n}}^{k+1}\left[\bar{\psi}_{n}\right] \leq Q_{n}{ }^{k}\left[\bar{\psi}_{n}\right] \leq \bar{\psi}_{n}$ holds, $\underline{\psi}_{n} \leq Q_{n}\left[\underline{\psi}_{n}\right] \leq Q_{n}{ }^{k+1}\left[\underline{\psi}_{n}\right] \leq Q_{n}{ }^{k+2}\left[\underline{\psi}_{n}\right] \leq$ $Q_{n}{ }^{k+2}\left[\bar{\psi}_{n}\right] \leq{Q_{n}}^{k+1}\left[\bar{\psi}_{n}\right] \leq Q_{n}\left[\bar{\psi}_{n}\right] \leq \bar{\psi}_{n}$ also holds. So, we have

$$
\underline{\psi}_{n} \leq Q_{n}{ }^{k}\left[\underline{\psi}_{n}\right] \leq Q_{n}{ }^{k+1}\left[\underline{\psi}_{n}\right] \leq Q_{n}{ }^{k+1}\left[\bar{\psi}_{n}\right] \leq Q_{n}{ }^{k}\left[\bar{\psi}_{n}\right] \leq \bar{\psi}_{n}
$$

for all $n \in \mathbb{N}$ and $k=0,1,2, \cdots$. We put $\phi_{n}:=\lim _{k \rightarrow \infty} Q_{n}{ }^{k}\left[\underline{\psi}_{n}\right] \in \mathcal{M}$. Then,

$$
\underline{\psi}_{n} \leq \phi_{n} \leq \bar{\psi}_{n}
$$

holds for all $n \in \mathbb{N}$. By Step 3, we also have

$$
Q_{n}\left[\phi_{n}\right]=\phi_{n}
$$

for all $n \in \mathbb{N}$. 
[Step 5] We take $N_{0} \in \mathbb{N}$ such that

$$
0 \leq \bar{\psi}\left(-N_{0}\right)<\alpha<\underline{\psi}\left(+N_{0}\right) \leq 1
$$

holds. Then, because $\phi_{n}\left(-\left(n+\frac{\bar{c}-\underline{c}}{2}+N_{0}\right)\right) \leq \bar{\psi}\left(-N_{0}\right)$ and $\psi\left(+N_{0}\right) \leq \phi_{n}(+(n+$ $\left.\frac{\bar{c}-\underline{c}}{2}+N_{0}\right)$ ) hold from (3.5), for any $n \in \mathbb{N}$, there exist constants $y_{n}$ and $z_{n}$ such that

$$
\begin{gathered}
\phi_{n}\left(y_{n}\right) \leq \frac{\bar{\psi}\left(-N_{0}\right)+\alpha}{2} \leq \lim _{h \downarrow+0} \phi_{n}\left(y_{n}+h\right), \\
\phi_{n}\left(z_{n}\right) \leq \frac{\alpha+\underline{\psi}\left(+N_{0}\right)}{2} \leq \lim _{h \downarrow+0} \phi_{n}\left(z_{n}+h\right)
\end{gathered}
$$

and

$$
-\left(n+\frac{\bar{c}-\underline{c}}{2}+N_{0}\right) \leq y_{n} \leq z_{n} \leq+\left(n+\frac{\bar{c}-\underline{c}}{2}+N_{0}\right)
$$

hold. As we put functions

$$
\phi_{-, n}(\cdot):=\phi_{n}\left(\cdot+y_{n}\right) \in \mathcal{M}
$$

and

$$
\phi_{+, n}(\cdot):=\phi_{n}\left(\cdot+z_{n}\right) \in \mathcal{M}
$$

we have

$$
\phi_{-, n}(0) \leq \frac{\bar{\psi}\left(-N_{0}\right)+\alpha}{2} \leq \lim _{h \downarrow+0} \phi_{-, n}(h)
$$

and

$$
\phi_{+, n}(0) \leq \frac{\alpha+\underline{\psi}\left(+N_{0}\right)}{2} \leq \lim _{h \downarrow+0} \phi_{+, n}(h)
$$

By Helly's theorem and (3.8), there exist a subsequence $\{n(k)\}_{k \in \mathbb{N}} \subset \mathbb{N}$, two functions $\phi_{-}, \phi_{+}$, two constants $\xi_{-}$and $\xi_{+}$such that the two equalities

$$
\phi_{-}(x)=\lim _{k \rightarrow \infty} \phi_{-, n(k)}(x) \in \mathcal{M}
$$

and

$$
\phi_{+}(x)=\lim _{k \rightarrow \infty} \phi_{+, n(k)}(x) \in \mathcal{M}
$$

hold almost everywhere in $x$ and the two equalities

$$
\xi_{-}=\lim _{k \rightarrow \infty} \frac{y_{n(k)}}{n(k)} \in[-1,+1]
$$


and

$$
\xi_{+}=\lim _{k \rightarrow \infty} \frac{z_{n(k)}}{n(k)} \in[-1,+1]
$$

hold. From (3.9), (3.10) and (3.8), we have

$$
\begin{aligned}
& \phi_{-}(0) \leq \frac{\bar{\psi}\left(-N_{0}\right)+\alpha}{2} \leq \lim _{h \downarrow+0} \phi_{-}(h), \\
& \phi_{+}(0) \leq \frac{\alpha+\underline{\psi}\left(+N_{0}\right)}{2} \leq \lim _{h \downarrow+0} \phi_{+}(h)
\end{aligned}
$$

and

$$
-1 \leq \xi_{-} \leq \xi_{+} \leq+1
$$

[Step 6] We show the following: The two equalities

$$
\left(Q_{0}\left[\phi_{-}\right]\right)\left(x-c_{-}\right) \equiv \phi_{-}(x)
$$

and

$$
\left(Q_{0}\left[\phi_{+}\right]\right)\left(x-c_{+}\right) \equiv \phi_{+}(x)
$$

hold, where $c_{-}$and $c_{+}$are the constants defined by

$$
c_{-}:=\frac{\underline{c}+\bar{c}}{2}-\frac{\bar{c}-\underline{c}_{\xi}}{2} \xi_{-}
$$

and

$$
c_{+}:=\frac{\underline{c}+\bar{c}}{2}-\frac{\bar{c}-\underline{c}}{2} \xi_{+} .
$$

Further, the inequality

$$
\underline{c} \leq c_{+} \leq c_{-} \leq \bar{c}
$$

holds.

From (3.2) and (3.12), we see

$$
\lim _{k \rightarrow \infty}\left(\rho_{n(k)}\left(x+y_{n(k)}\right)-y_{n(k)}\right)=x-c_{-}
$$

for all $x \in \mathbb{R}$. Hence, by Lemma 12 of [18], (3.11) and $\left(A_{n}\left[\phi_{n}\right]\right)\left(x+y_{n}\right)=$ $\phi_{n}\left(\rho_{n}\left(x+y_{n}\right)\right)=\phi_{-, n}\left(\rho_{n}\left(x+y_{n}\right)-y_{n}\right)$, we have

$$
\lim _{k \rightarrow \infty}\left(A_{n(k)}\left[\phi_{n(k)}\right]\right)\left(x+y_{n(k)}\right)=\phi_{-}\left(x-c_{-}\right)
$$


for all continuous points $x \in \mathbb{R}$ of $\phi_{-}\left(x-c_{-}\right)$. From (3.6),

$$
\begin{gathered}
\phi_{-, n}(x)=\phi_{n}\left(x+y_{n}\right)=\left(Q_{n}\left[\phi_{n}\right]\right)\left(x+y_{n}\right) \\
=\left(Q_{0}\left[A_{n}\left[\phi_{n}\right]\right]\right)\left(x+y_{n}\right)=\left(Q_{0}\left[\left(A_{n}\left[\phi_{n}\right]\right)\left(\cdot+y_{n}\right)\right]\right)(x)
\end{gathered}
$$

holds for all $n \in \mathbb{N}$ and $x \in \mathbb{R}$. By Proposition 9 of [18], (3.19), (3.20) and (3.11), we obtain

$$
\begin{gathered}
\left(Q_{0}\left[\phi_{-}\right]\right)\left(x-c_{-}\right)=\left(Q_{0}\left[\phi_{-}\left(\cdot-c_{-}\right)\right]\right)(x) \\
=\lim _{k \rightarrow \infty}\left(Q_{0}\left[\left(A_{n(k)}\left[\phi_{n(k)}\right]\right)\left(\cdot+y_{n(k)}\right)\right]\right)(x) \\
=\lim _{k \rightarrow \infty} \phi_{-, n(k)}(x)=\phi_{-}(x) .
\end{gathered}
$$

Almost similarly as (3.16), we also obtain (3.17). Further, (3.18) follows from (3.1) and (3.15).

[Step 7] By Proposition 9 of [18] and (3.16), we have

$$
\begin{gathered}
Q_{0}\left[\phi_{-}(-\infty)\right]=\left(Q_{0}\left[\phi_{-}(-\infty)\right]\right)(0)=\lim _{k \rightarrow \infty}\left(Q_{0}\left[\phi_{-}(\cdot-k)\right]\right)(0) \\
=\lim _{k \rightarrow \infty}\left(Q_{0}\left[\phi_{-}\right]\right)(-k)=\left(Q_{0}\left[\phi_{-}\right]\right)(-\infty)=\phi_{-}(-\infty) .
\end{gathered}
$$

Almost similarly, we also have $Q_{0}\left[\phi_{-}(+\infty)\right]=\phi_{-}(+\infty), Q_{0}\left[\phi_{+}(-\infty)\right]=\phi_{+}(-\infty)$ and $Q_{0}\left[\phi_{+}(+\infty)\right]=\phi_{+}(+\infty)$ by Proposition 9 of [18], (3.16) and (3.17). From (3.7), (3.13) and (3.14), we see $0 \leq \phi_{-}(-\infty)<\alpha, 0<\phi_{-}(+\infty) \leq 1,0 \leq$ $\phi_{+}(-\infty)<1$ and $\alpha<\phi_{+}(+\infty) \leq 1$. Therefore, because $\{\gamma \in \mathbb{R} \mid 0 \leq \gamma \leq$ $\left.1, Q_{0}[\gamma]=\gamma\right\}=\{0, \alpha, 1\}$ holds from Hypothesis 2 (iv), we obtain

$$
\begin{gathered}
\phi_{-}(-\infty)=0, \\
\phi_{-}(+\infty)=\alpha \text { or } 1, \\
\phi_{+}(-\infty)=0 \text { or } \alpha
\end{gathered}
$$

and

$$
\phi_{+}(+\infty)=1 .
$$

[Step 8] We show that $\phi_{-}(+\infty) \neq \alpha$ or $\phi_{+}(-\infty) \neq \alpha$ holds. Suppose that $\phi_{-}(+\infty)=\alpha$ and $\phi_{+}(-\infty)=\alpha$ hold. Then, from Hypothesis 3, (3.16), (3.17), (3.21) and (3.24), we have $c_{-}<c_{+}$. It is a contradiction with (3.18). So, we 
see that $\phi_{-}(+\infty) \neq \alpha$ or $\phi_{+}(-\infty) \neq \alpha$ holds. Hence, from (3.22) and (3.23), we also see that

$$
\phi_{-}(+\infty)=1 \text { or } \phi_{+}(-\infty)=0
$$

holds. When $\phi_{-}(+\infty)=1$, we obtain the conclusion of Theorem 4 with $c:=c_{-}$ and $\phi:=\phi_{-}$because of (3.18), (3.21) and (3.16). When $\phi_{+}(-\infty)=0$, we obtain it with $c:=c_{+}$and $\phi:=\phi_{+}$because of (3.18), (3.24) and (3.17).

Proof of Corollary 5.

We put functions $\underline{\psi}$ and $\bar{\psi} \in \mathcal{M}$ as

$$
\underline{\psi}(x)=0 \quad(x \leq 0), \quad \underline{\psi}(x)=\frac{\alpha+1}{2} \quad(0<x)
$$

and

$$
\bar{\psi}(x)=\frac{\alpha}{2} \quad(x \leq-1), \quad \bar{\psi}(x)=1 \quad(-1<x) .
$$

Then, by Proposition 9 of [18] and Hypothesis 2 (iv), we have

$$
\begin{gathered}
\left(Q_{0}[\underline{\psi}]\right)(+\infty)=\lim _{k \rightarrow \infty}\left(Q_{0}[\underline{\psi}]\right)(k)=\lim _{k \rightarrow \infty}\left(Q_{0}[\underline{\psi}(\cdot+k)]\right)(0) \\
=\left(Q_{0}[\underline{\psi}(+\infty)]\right)(0)=Q_{0}[\underline{\psi}(+\infty)]>\underline{\psi}(+\infty) .
\end{gathered}
$$

Almost similarly, we also have $\left(Q_{0}[\bar{\psi}]\right)(-\infty)<\bar{\psi}(-\infty)$. Hence, there exist constants $\underline{c}$ and $\bar{c}$ such that $\underline{\psi}(+\infty) \leq\left(Q_{0}[\underline{\psi}]\right)(-\underline{c})$ and $\left(Q_{0}[\bar{\psi}]\right)(-1-\bar{c}) \leq$ $\bar{\psi}(-\infty)$ hold. So, because $\underline{\psi}(\bar{x}) \leq\left(Q_{0}[\underline{\psi}]\right)(x-\underline{c})$ and $\left(Q_{0}[\bar{\psi}]\right)(x-\bar{c}) \leq \bar{\psi}(x)$ also hold for all $x \in \mathbb{R}$, in virtue of Theorem 4 , we obtain the conclusion of Corollary 5 .

The following lemma follows from Theorem 5 of [18]:

Lemma 9. Let $Q^{t}$ be a map from $\mathcal{M}$ to $\mathcal{M}$ for $t \in[0,+\infty)$. Suppose the map $Q^{t}$ satisfies Hypotheses 2 for all $t \in(0,+\infty)$, and the family $Q:=$ $\left\{Q^{t}\right\}_{t \in[0,+\infty)}$ Hypotheses 6 . Then, the following two hold:

(i) Let $\tau \in(0,+\infty)$ and $c_{-} \in \mathbb{R}$. Suppose there exists $\phi_{-} \in \mathcal{M}$ with $\left(Q^{\tau}\left[\phi_{-}\right]\right)\left(x-c_{-} \tau\right) \equiv \phi_{-}(x), \phi_{-}(-\infty)=0$ and $\phi_{-}(+\infty)=\alpha$. Then, there exists $\varphi_{-} \in \mathcal{M}$ with $\varphi_{-}(-\infty)=0$ and $\varphi_{-}(+\infty)=\alpha$ such that $\left(Q^{t}\left[\varphi_{-}\right]\right)\left(x-c_{-} t\right) \equiv$ $\varphi_{-}(x)$ holds for all $t \in[0,+\infty)$.

(ii) Let $\tau \in(0,+\infty)$ and $c_{+} \in \mathbb{R}$. Suppose there exists $\phi_{+} \in \mathcal{M}$ with $\left(Q^{\tau}\left[\phi_{+}\right]\right)\left(x-c_{+} \tau\right) \equiv \phi_{+}(x), \phi_{+}(-\infty)=\alpha$ and $\phi_{+}(+\infty)=1$. Then, there exists $\varphi_{+} \in \mathcal{M}$ with $\varphi_{+}(-\infty)=\alpha$ and $\varphi_{+}(+\infty)=1$ such that $\left(Q^{t}\left[\varphi_{+}\right]\right)\left(x-c_{+} t\right) \equiv$ $\varphi_{+}(x)$ holds for all $t \in[0,+\infty)$. 
Proof. We show (i). Put a set as

$$
\mathcal{M}_{-}:=\{u \mid u \text { is a monotone nondecreasing }
$$

and left continuous function on $\mathbb{R}$ with $0 \leq u \leq \alpha\}$.

Put two maps $R_{-}: \mathcal{M} \rightarrow \mathcal{M}_{-}$and $S_{-}: \mathcal{M}_{-} \rightarrow \mathcal{M}$ as

$$
\left(R_{-}[u]\right)(x):=\alpha\left(1-\lim _{h \downarrow+0} u(-x+h)\right)
$$

and

$$
\left(S_{-}\left[u_{-}\right]\right)(x):=-\frac{1}{\alpha}\left(\lim _{h \downarrow+0} u_{-}(-x+h)\right)+1 .
$$

So, the maps $R_{-}$and $S_{-}$are inverse in each other. In virtue of Hypotheses 2 (ii) and (iv), we can define a map $Q_{-}^{t}: \mathcal{M} \rightarrow \mathcal{M}$ by

$$
Q_{-}^{t}:=S_{-} \circ Q^{t} \circ R_{-}
$$

for $t \in[0,+\infty)$. Then, in virtue of Hypotheses 2 and $6, Q_{-}:=\left\{Q_{-}^{t}\right\}_{t \in[0,+\infty)}$ satisfies the assumption of Theorem 5 of [18]. Hence, Theorem 5 of [18] works for the semiflow $Q_{-}$. Let $\tilde{\phi}_{-}:=S_{-}\left[\phi_{-}\right] \in \mathcal{M}$. Then, $\left(Q_{-}^{\tau}\left[\tilde{\phi}_{-}\right]\right)\left(x-c_{-} \tau\right) \equiv$ $\tilde{\phi}_{-}(x), \tilde{\phi}_{-}(-\infty)=0$ and $\tilde{\phi}_{-}(+\infty)=1$ hold. Therefore, by Theorem 5 of [18], there exists $\tilde{\varphi}_{-} \in \mathcal{M}$ with $\tilde{\varphi}_{-}(-\infty)=0$ and $\tilde{\varphi}_{-}(+\infty)=1$ such that $\left(Q_{-}^{t}\left[\tilde{\varphi}_{-}\right]\right)\left(x-c_{-} t\right) \equiv \tilde{\varphi}_{-}(x)$ holds for all $t \in[0,+\infty)$. So, as we put $\varphi_{-}:=$ $R_{-}\left[\tilde{\varphi}_{-}\right] \in \mathcal{M}_{-}$, we obtain the conclusion of (i).

We show (ii). Put a set as

$$
\mathcal{M}_{+}:=\{u \mid u \text { is a monotone nondecreasing }
$$

and left continuous function on $\mathbb{R}$ with $\alpha \leq u \leq 1\}$.

Put two maps $R_{+}: \mathcal{M} \rightarrow \mathcal{M}_{+}$and $S_{+}: \mathcal{M}_{+} \rightarrow \mathcal{M}$ as

$$
\left(R_{+}[u]\right)(x):=(1-\alpha) u(x)+\alpha
$$

and

$$
\left(S_{+}\left[u_{+}\right]\right)(x):=\frac{1}{1-\alpha}\left(u_{+}(x)-\alpha\right) .
$$

Then, almost similarly as (i), we can obtain the conclusion of (ii).

Proof of Theorem 8.

[Step 1] First, we show that the map $Q^{\tau}$ satisfies Hypothesis 3 for all $\tau \in(0,+\infty)$. Let a positive constant $\tau$, two constants $c_{-}$and $c_{+}$and two functions $\phi_{-}$and $\phi_{+} \in \mathcal{M}$ satisfy $\left(Q^{\tau}\left[\phi_{-}\right]\right)\left(x-c_{-}\right) \equiv \phi_{-}(x), \phi_{-}(-\infty)=0$, 
$\phi_{-}(+\infty)=\alpha,\left(Q^{\tau}\left[\phi_{+}\right]\right)\left(x-c_{+}\right) \equiv \phi_{+}(x), \phi_{+}(-\infty)=\alpha$ and $\phi_{+}(+\infty)=1$. Then, by Lemma 9 , there exist $\varphi_{-}$and $\varphi_{+} \in \mathcal{M}$ with $\varphi_{-}(-\infty)=0, \varphi_{-}(+\infty)=$ $\alpha, \varphi_{+}(-\infty)=\alpha$ and $\varphi_{+}(+\infty)=1$ such that $\left(Q^{t}\left[\varphi_{-}\right]\right)\left(x-\frac{c_{-}}{\tau} t\right) \equiv \varphi_{-}(x)$ and $\left(Q^{t}\left[\varphi_{+}\right]\right)\left(x-\frac{c_{+}}{\tau} t\right) \equiv \varphi_{+}(x)$ hold for all $t \in[0,+\infty)$. Hence, in virtue of Hypothesis 7 , we see $\frac{c_{-}}{\tau}<\frac{c_{+}}{\tau}$ and so $c_{-}<c_{+}$. Therefore, the map $Q^{t}$ satisfies Hypothesis 3 for all $t \in(0,+\infty)$.

Then, by Theorem 4 , for any $n \in \mathbb{N}$, there exist $c_{n} \in[\underline{c}, \bar{c}]$ and $\phi_{n} \in \mathcal{M}$ with $\phi_{n}(-\infty)=0$ and $\phi_{n}(+\infty)=1$ such that $\left(Q^{\frac{1}{2^{n}}}\left[\phi_{n}\right]\right)\left(x-\frac{c_{n}}{2^{n}}\right) \equiv \phi_{n}(x)$ holds. Then, for any $n \in \mathbb{N}$, there exist constants $y_{n}$ and $z_{n}$ such that

$$
\phi_{n}\left(y_{n}\right) \leq \frac{\alpha}{2} \leq \lim _{h \downarrow+0} \phi_{n}\left(y_{n}+h\right)
$$

and

$$
\phi_{n}\left(z_{n}\right) \leq \frac{\alpha+1}{2} \leq \lim _{h \downarrow+0} \phi_{n}\left(z_{n}+h\right)
$$

hold. As we put functions

$$
\phi_{-, n}(\cdot):=\phi_{n}\left(\cdot+y_{n}\right) \in \mathcal{M}
$$

and

$$
\phi_{+, n}(\cdot):=\phi_{n}\left(\cdot+z_{n}\right) \in \mathcal{M},
$$

we have

$$
\begin{gathered}
\left(Q^{\frac{1}{2^{n}}}\left[\phi_{-, n}\right]\right)\left(x-\frac{c_{n}}{2^{n}}\right) \equiv \phi_{-, n}(x), \\
\left(Q^{\frac{1}{2^{n}}}\left[\phi_{+, n}\right]\right)\left(x-\frac{c_{n}}{2^{n}}\right) \equiv \phi_{+, n}(x), \\
\phi_{-, n}(0) \leq \frac{\alpha}{2} \leq \lim _{h \downarrow+0} \phi_{-, n}(h)
\end{gathered}
$$

and

$$
\phi_{+, n}(0) \leq \frac{\alpha+1}{2} \leq \lim _{h \downarrow+0} \phi_{+, n}(h) .
$$

By Helly's theorem, there exist a subsequence $\{n(k)\}_{k \in \mathbb{N}} \subset \mathbb{N}$, two functions $\phi_{-}, \phi_{+}$and a constant $c$ such that the two equalities

$$
\phi_{-}(x)=\lim _{k \rightarrow \infty} \phi_{-, n(k)}(x) \in \mathcal{M}
$$

and

$$
\phi_{+}(x)=\lim _{k \rightarrow \infty} \phi_{+, n(k)}(x) \in \mathcal{M}
$$


hold almost everywhere in $x$ and the equality

$$
c=\lim _{k \rightarrow \infty} c_{n(k)} \in[\underline{c}, \bar{c}]
$$

holds. From (3.26) and (3.27), we have

$$
\phi_{-}(0) \leq \frac{\alpha}{2} \leq \lim _{h \downarrow+0} \phi_{-}(h)
$$

and

$$
\phi_{+}(0) \leq \frac{\alpha+1}{2} \leq \lim _{h \downarrow+0} \phi_{+}(h) .
$$

[Step 2] We show the following: The two equalities

$$
\left(Q^{t}\left[\phi_{-}\right]\right)(x-c t) \equiv \phi_{-}(x)
$$

and

$$
\left(Q^{t}\left[\phi_{+}\right]\right)(x-c t) \equiv \phi_{+}(x)
$$

hold for all $t \in[0,+\infty)$.

Let $n_{0} \in \mathbb{N}$ and $m_{0} \in \mathbb{N}$. As $k \in \mathbb{N}$ is sufficiently large,

$$
\begin{aligned}
& \left(Q^{\frac{m_{0}}{2^{n_{0}}}}\left[\phi_{-, n(k)}\right]\right)\left(x-c_{n(k)} \frac{m_{0}}{2^{n_{0}}}\right) \\
& =\left(\left(Q^{\frac{1}{2^{n(k)}}}\right)^{m_{0} 2^{n(k)-n_{0}}}\left[\phi_{-, n(k)}\right]\right)\left(x-\frac{c_{n(k)}}{2^{n(k)}} m_{0} 2^{n(k)-n_{0}}\right)=\phi_{-, n(k)}(x)
\end{aligned}
$$

holds because of $n(k) \geq n_{0}$ and (3.25). Hence, by (3.28), (3.29), Lemma 12 of [18] and Proposition 9 of [18], we obtain

$$
\left(Q^{\frac{m_{0}}{2^{n_{0}}}}\left[\phi_{-}\right]\right)\left(x-c \frac{m_{0}}{2^{n_{0}}}\right)=\phi_{-}(x)
$$

for all $n_{0} \in \mathbb{N}$ and $m_{0} \in \mathbb{N}$.

Let $t \in[0,+\infty)$. Then, by (3.34), there exists a sequence $\left\{t_{k}\right\}_{k \in \mathbb{N}} \subset$ $[0,+\infty)$ with $\lim _{k \rightarrow \infty} t_{k}=0$ such that $\left(Q^{t+t_{k}}\left[\phi_{-}\right]\right)\left(x-c\left(t+t_{k}\right)\right)=\phi_{-}(x)$ holds for all $k \in \mathbb{N}$. So, by $\left(Q^{t_{k}}\left[\left(Q^{t}\left[\phi_{-}\right]\right)(\cdot-c t)\right]\right)\left(x-c t_{k}\right)=\left(Q^{t+t_{k}}\left[\phi_{-}\right]\right)\left(x-c\left(t+t_{k}\right)\right)$ and Lemma 14 of [18], we obtain $\left(Q^{t}\left[\phi_{-}\right]\right)(x-c t)=\phi_{-}(x)$.

Almost similarly as (3.32), we also obtain (3.33).

[Step 3] By Proposition 9 of [18] and (3.32), we have

$$
\begin{gathered}
Q^{t}\left[\phi_{-}(-\infty)\right]=\left(Q^{t}\left[\phi_{-}(-\infty)\right]\right)(0)=\lim _{k \rightarrow \infty}\left(Q^{t}\left[\phi_{-}(\cdot-k)\right]\right)(0) \\
=\lim _{k \rightarrow \infty}\left(Q^{t}\left[\phi_{-}\right]\right)(-k)=\left(Q^{t}\left[\phi_{-}\right]\right)(-\infty)=\phi_{-}(-\infty) .
\end{gathered}
$$


Almost similarly, we also have $Q^{t}\left[\phi_{-}(+\infty)\right]=\phi_{-}(+\infty), Q^{t}\left[\phi_{+}(-\infty)\right]=\phi_{+}(-\infty)$ and $Q^{t}\left[\phi_{+}(+\infty)\right]=\phi_{+}(+\infty)$ by Proposition 9 of [18], (3.32) and (3.33). From (3.30) and (3.31), we see $0 \leq \phi_{-}(-\infty)<\alpha, 0<\phi_{-}(+\infty) \leq 1,0 \leq \phi_{+}(-\infty)<$ 1 and $\alpha<\phi_{+}(+\infty) \leq 1$. Therefore, from Hypothesis 2 (iv), we obtain

$$
\begin{gathered}
\phi_{-}(-\infty)=0, \\
\phi_{-}(+\infty)=\alpha \text { or } 1, \\
\phi_{+}(-\infty)=0 \text { or } \alpha
\end{gathered}
$$

and

$$
\phi_{+}(+\infty)=1 .
$$

[Step 4] We show that $\phi_{-}(+\infty) \neq \alpha$ or $\phi_{+}(-\infty) \neq \alpha$ holds. Suppose that $\phi_{-}(+\infty)=\alpha$ and $\phi_{+}(-\infty)=\alpha$ hold. Then, from Hypothesis 3, (3.32), (3.33), (3.35) and (3.38), we have the contradiction $c<c$. So, we see that $\phi_{-}(+\infty) \neq \alpha$ or $\phi_{+}(-\infty) \neq \alpha$ holds. Hence, from (3.36) and (3.37), we also see that

$$
\phi_{-}(+\infty)=1 \text { or } \phi_{+}(-\infty)=0
$$

holds. When $\phi_{-}(+\infty)=1$, we obtain the conclusion of Theorem 8 with $\phi:=$ $\phi_{-}$. When $\phi_{+}(-\infty)=0$, we obtain it with $\phi:=\phi_{+}$.

\section{$\S 4 . \quad$ Proof of Theorem 1}

We recall that $\mu$ is a Borel-measure on $\mathbb{R}$ with $\mu(\mathbb{R})=1, f$ is a Lipschitz continuous function on $\mathbb{R}$ and satisfies $f(0)=f(\alpha)=f(1)=0, f<0$ in $(0, \alpha)$ and $f>0$ in $(\alpha, 1)$ for some constant $\alpha \in(0,1)$ and the set $\mathcal{M}$ has been defined at the beginning of Section 2. Then, in virtue of Lemma 15 of [18], Lemma 16 of [18] and Proposition 18 of [18], $Q^{t}(t \in(0,+\infty))$ satisfies Hypotheses 2 and $Q$ Hypotheses 6 for the semiflow $Q=\left\{Q^{t}\right\}_{t \in[0,+\infty)}$ on $\mathcal{M}$ generated by (1.1). So, if we would confirm that this semiflow on $\mathcal{M}$ satisfies Hypothesis 7 , then we could make Theorem 8 of Section 2 work. In this section, we confirm it when $f^{\prime}(\alpha)>0$ and $\mu(\{0\}) \neq 1$ hold and construct sub and super-solutions to prove Theorem 1.

First, we consider the linear equation

$$
v_{t}=\hat{\mu} * v .
$$


It generates a flow on the Banach space $B C(\mathbb{R})$ when $\hat{\mu}(\mathbb{R})<+\infty$. Here, $B C(\mathbb{R})$ denote the set of bounded and continuous functions on $\mathbb{R}$. We have the following for this flow on $B C(\mathbb{R})$ :

Proposition 10. Let $\hat{\mu}$ be a Borel-measure on $\mathbb{R}$ with $\hat{\mu}(\mathbb{R})<+\infty$. Let $\hat{P}: B C(\mathbb{R}) \rightarrow B C(\mathbb{R})$ be the time 1 map of the flow on $B C(\mathbb{R})$ generated by the linear equation (4.1). Then, there exists a Borel-measure $\hat{\nu}$ on $\mathbb{R}$ with $\hat{\nu}(\mathbb{R})<+\infty$ such that

$$
\hat{P}[v]=\hat{\nu} * v
$$

holds for all $v \in B C(\mathbb{R})$. Further, the equality

$$
\log \int_{y \in \mathbb{R}} e^{\lambda y} d \hat{\nu}(y)=\int_{y \in \mathbb{R}} e^{\lambda y} d \hat{\mu}(y)
$$

holds for all $\lambda \in \mathbb{R}$.

Proof. From Lemma 24 of [18], there exists a Borel-measure $\hat{\nu}$ on $\mathbb{R}$ with $\hat{\nu}(\mathbb{R})<+\infty$ such that

$$
\hat{P}[v]=\hat{\nu} * v
$$

holds for all $v \in B C(\mathbb{R})$. Further, from Lemma 24 of [18], if $v$ is a nonnegative, bounded and continuous function on $\mathbb{R}$, then the inequality

$$
\hat{\mu} * v \leq \hat{\nu} * v
$$

holds. So, because

$$
\begin{aligned}
& \int_{y \in \mathbb{R}} e^{\lambda y} d \hat{\mu}(y)=\lim _{n \rightarrow \infty} \int_{y \in \mathbb{R}} \min \left\{e^{\lambda y}, n\right\} d \hat{\mu}(y)=\lim _{n \rightarrow \infty}\left(\hat{\mu} * \min \left\{e^{-\lambda x}, n\right\}\right)(0) \\
& \leq \lim _{n \rightarrow \infty}\left(\hat{\nu} * \min \left\{e^{-\lambda x}, n\right\}\right)(0)=\lim _{n \rightarrow \infty} \int_{y \in \mathbb{R}} \min \left\{e^{\lambda y}, n\right\} d \hat{\nu}(y)=\int_{y \in \mathbb{R}} e^{\lambda y} d \hat{\nu}(y)
\end{aligned}
$$

holds, $\int_{y \in \mathbb{R}} e^{\lambda y} d \hat{\mu}(y)=+\infty$ implies $\int_{y \in \mathbb{R}} e^{\lambda y} d \hat{\nu}(y)=+\infty$. Therefore, it is sufficient if we show that the equality (4.2) holds when

$$
\int_{y \in \mathbb{R}} e^{\lambda y} d \hat{\mu}(y)<+\infty .
$$

Let $\lambda \in \mathbb{R}$. Suppose (4.4).

Let $X_{\lambda}$ denote the set of continuous functions $u$ on $\mathbb{R}$ with $\sup _{x \in \mathbb{R}} \frac{|u(x)|}{1+e^{-\lambda x}}<$ $+\infty$. Then, $X_{\lambda}$ is a Banach space with the norm $\|u\|_{X_{\lambda}}:=\sup _{x \in \mathbb{R}} \frac{|u(x)|}{1+e^{-\lambda x}}$. Let $u \in X_{\lambda}$. Then, for any $x$ and $y \in \mathbb{R}$, we have

$$
\sup _{h \in[-1,+1]}|u((x+h)-y)-u(x-y)|
$$




$$
\begin{aligned}
& \leq\|u\|_{X_{\lambda}} \sup _{h \in[-1,+1]}\left(\left(1+e^{-\lambda((x+h)-y)}\right)+\left(1+e^{-\lambda(x-y)}\right)\right) \\
\leq & \|u\|_{X_{\lambda}}\left(\sup _{h \in[-1,+1]}\left(\left(1+e^{-\lambda(x+h)}\right)+\left(1+e^{-\lambda x}\right)\right)\right)\left(1+e^{\lambda y}\right) .
\end{aligned}
$$

Hence, from (4.4), the function $\hat{\mu} * u$ is continuous. Because

$$
\begin{gathered}
\sup _{x \in \mathbb{R}} \frac{|(\hat{\mu} * u)(x)|}{1+e^{-\lambda x}} \leq \sup _{x \in \mathbb{R}} \int_{y \in \mathbb{R}} \frac{|u(x-y)|}{1+e^{-\lambda(x-y)}}\left(1+e^{\lambda y}\right) d \hat{\mu}(y) \\
\leq\left(\int_{y \in \mathbb{R}}\left(1+e^{\lambda y}\right) d \hat{\mu}(y)\right)\|u\|_{X_{\lambda}}
\end{gathered}
$$

also holds, the map $u \mapsto \hat{\mu} * u$ is a bounded and linear operator in the Banach space $X_{\lambda}$. Let $\hat{P}_{\lambda}: X_{\lambda} \rightarrow X_{\lambda}$ be the time 1 map of the flow on $X_{\lambda}$ generated by the linear equation (4.1).

Suppose $\lambda>0$. Let $\bar{\lambda} \in(0, \lambda)$. Then, we see

$$
\begin{aligned}
& \lim _{n \rightarrow \infty}\left\|\min \left\{e^{-\bar{\lambda} x}, n\right\}-e^{-\bar{\lambda} x}\right\|_{X_{\lambda}} \\
\leq & \lim _{n \rightarrow \infty} \sup _{x \in\left(-\infty,-\frac{1}{\lambda} \log n\right)} \frac{e^{-\bar{\lambda} x}}{1+e^{-\lambda x}} \\
\leq & \lim _{n \rightarrow \infty} \sup _{x \in\left(-\infty,-\frac{1}{\lambda} \log n\right)} e^{(\lambda-\bar{\lambda}) x}=0 .
\end{aligned}
$$

The function $v(t, x):=e^{\left(\int_{y \in \mathbb{R}} e^{\bar{\lambda} y} d \hat{\mu}(y)\right) t-\bar{\lambda} x}$ is a solution to (4.1) in the phase space $X_{\lambda}$. Hence, by (4.3) and (4.5),

$$
\begin{gathered}
\int_{y \in \mathbb{R}} e^{\bar{\lambda} y} d \hat{\nu}(y)=\lim _{n \rightarrow \infty} \int_{y \in \mathbb{R}} \min \left\{e^{\bar{\lambda} y}, n\right\} d \hat{\nu}(y) \\
=\lim _{n \rightarrow \infty}\left(\hat{\nu} * \min \left\{e^{-\bar{\lambda} x}, n\right\}\right)(0)=\lim _{n \rightarrow \infty}\left(\hat{P}\left[\min \left\{e^{-\bar{\lambda} x}, n\right\}\right]\right)(0) \\
=\lim _{n \rightarrow \infty}\left(\hat{P}_{\lambda}\left[\min \left\{e^{-\bar{\lambda} x}, n\right\}\right]\right)(0)=\left(\hat{P}_{\lambda}\left[e^{-\bar{\lambda} x}\right]\right)(0)=e^{\int_{y \in \mathbb{R}} e^{\bar{\lambda} y} d \hat{\mu}(y)}
\end{gathered}
$$

holds for all $\bar{\lambda} \in(0, \lambda)$. So, we have

$$
\int_{y \in \mathbb{R}} e^{\lambda y} d \hat{\nu}(y)=\lim _{\bar{\lambda} \uparrow \lambda} \int_{y \in \mathbb{R}} e^{\bar{\lambda} y} d \hat{\nu}(y)=\lim _{\bar{\lambda} \uparrow \lambda} e^{\int_{y \in \mathbb{R}} e^{\bar{\lambda} y} d \hat{\mu}(y)}=e^{\int_{y \in \mathbb{R}} e^{\lambda y} d \hat{\mu}(y)} .
$$

When $\lambda<0$, we could also prove it almost similarly as $\lambda>0$.

Because $e^{\left(\int_{y \in \mathbb{R}} 1 d \hat{\mu}(y)\right) t}$ is a solution to (4.1), from (4.3), we see

$$
\int_{y \in \mathbb{R}} 1 d \hat{\nu}(y)=(\hat{\nu} * 1)(0)=(\hat{P}[1])(0)=e^{\int_{y \in \mathbb{R}} 1 d \hat{\mu}(y)} .
$$


So, the equality (4.2) also holds when $\lambda=0$.

In Section 6 of [18], the author has recalled a method to estimate the spreading speeds in monostable systems by Weinberger [17]. Combining Proposition 10 with the method, we have the following:

Lemma 11. Suppose a constant $\sigma$ satisfies $0<\sigma<f^{\prime}(\alpha)$. Then, the following two hold:

(i) Let $c_{-} \in \mathbb{R}$. Let $\phi_{-}$be a monotone function on $\mathbb{R}$ with $\phi_{-}(-\infty)=0$ and $\phi_{-}(+\infty)=\alpha$. Suppose $u_{-}(t, x):=\phi_{-}\left(x+c_{-} t\right)$ is a solution to (1.1). Then,

$$
\inf _{\lambda_{-}>0} \frac{\int_{y \in \mathbb{R}} e^{\lambda_{-} y} d \mu(y)-1+\sigma}{\lambda_{-}} \leq-c_{-}
$$

holds.

(ii) Let $c_{+} \in \mathbb{R}$. Let $\phi_{+}$be a monotone function on $\mathbb{R}$ with $\phi_{+}(-\infty)=\alpha$ and $\phi_{+}(+\infty)=1$. Suppose $u_{+}(t, x):=\phi_{+}\left(x+c_{+} t\right)$ is a solution to (1.1). Then,

$$
\inf _{\lambda_{+}>0} \frac{\int_{y \in \mathbb{R}} e^{-\lambda_{+} y} d \mu(y)-1+\sigma}{\lambda_{+}} \leq c_{+}
$$

holds.

Proof. $\quad[$ Step 1] In this step, we show (i).

We put a Borel-measure $\hat{\mu}:=\mu$ and a Lipschitz continuous function $\hat{f}(u):=-\frac{1}{\alpha} f(-\alpha(u-1))$. Then, we see $\hat{f}(0)=\hat{f}(1)=0, f>0$ in $(0,1)$ and

$$
0<\sigma<f^{\prime}(\alpha)=\hat{f}^{\prime}(0)
$$

Further, we put a monotone function $\hat{\phi}(z):=-\frac{1}{\alpha} \phi_{-}(z)+1$ with $\hat{\phi}(-\infty)=1$ and $\hat{\phi}(+\infty)=0$. Then, the function $u(t, x):=\hat{\phi}\left(x+c_{-} t\right)$ is a solution to

$$
u_{t}=\hat{\mu} * u-u+\hat{f}(u) \text {. }
$$

Let $\hat{P}: B C(\mathbb{R}) \rightarrow B C(\mathbb{R})$ be the time 1 map of the flow on $B C(\mathbb{R})$ generated by the linear equation (4.1). Then, by Proposition 10, there exists a Borel-measure $\hat{\nu}$ on $\mathbb{R}$ with $\hat{\nu}(\mathbb{R})<+\infty$ such that

$$
\hat{P}[v]=\hat{\nu} * v
$$

holds for all $v \in B C(\mathbb{R})$. Further, the equality

$$
\log \int_{y \in \mathbb{R}} e^{\lambda y} d \hat{\nu}(y)=\int_{y \in \mathbb{R}} e^{\lambda y} d \hat{\mu}(y)
$$


holds for all $\lambda \in \mathbb{R}$. Let $\tilde{P}: B C(\mathbb{R}) \rightarrow B C(\mathbb{R})$ be the time 1 map of the flow on $B C(\mathbb{R})$ generated by the linear equation

$$
v_{t}=\hat{\mu} * v-v+\sigma v .
$$

Then, from (4.8) and (4.9), as $\tilde{\nu}$ is the Borel-measure on $\mathbb{R}$ defined by

$$
\tilde{\nu}:=e^{-1+\sigma} \hat{\nu},
$$

holds for all $v \in B C(\mathbb{R})$ and

$$
\log \int_{y \in \mathbb{R}} e^{\lambda y} d \tilde{\nu}(y)=\int_{y \in \mathbb{R}} e^{\lambda y} d \hat{\mu}(y)-1+\sigma
$$

holds for all $\lambda \in \mathbb{R}$. Because $\tilde{\nu}(\mathbb{R})=(\tilde{\nu} * 1)(0)=(\tilde{P}[1])(0)=e^{\sigma}$ holds from (4.10) and $\hat{\mu}(\mathbb{R})=1$, we also have

$$
1<\tilde{\nu}(\mathbb{R})<+\infty .
$$

Let $\tilde{Q}_{0}: \mathcal{B} \rightarrow \mathcal{B}$ be the time 1 map of the semiflow on $\mathcal{B}$ generated by the equation (4.7), where $\mathcal{B}$ is the set defined by

$$
\mathcal{B}:=\{u \mid u \text { is a continuous function on } \mathbb{R} \text { with } 0 \leq u \leq 1\} .
$$

Then, from Lemma 25 of [18], (4.6) and (4.10), there exists a positive constant $\varepsilon$ such that the inequality

$$
\tilde{\nu} * u=\tilde{P}[u] \leq \tilde{Q}_{0}[u]
$$

holds for all $u \in \mathcal{B}$ with $u \leq \varepsilon$. Therefore, by Proposition 23 of [18] and (4.12), we obtain the inequality

$$
\inf _{\lambda>0} \frac{1}{\lambda} \log \int_{y \in \mathbb{R}} e^{\lambda y} d \tilde{\nu}(y) \leq-c_{-} .
$$

So, from (4.11), we obtain

$$
\inf _{\lambda>0} \frac{\int_{y \in \mathbb{R}} e^{\lambda y} d \hat{\mu}(y)-1+\sigma}{\lambda} \leq-c_{-} .
$$

[Step 2] We show (ii). Let $\hat{\mu}$ be the Borel-measure on $\mathbb{R}$ such that

$$
\hat{\mu}((-\infty, y))=\mu((-y,+\infty))
$$


holds for all $y \in \mathbb{R}$. We put a Lipschitz continuous function $\hat{f}(u):=\frac{1}{1-\alpha} f((1-$ $\alpha) u+\alpha)$ and a monotone function $\hat{\phi}(z):=\frac{1}{1-\alpha}\left(\phi_{+}(-z)-\alpha\right)$ with $\hat{\phi}(-\infty)=1$ and $\hat{\phi}(+\infty)=0$. Then, the function $u(t, x):=\hat{\phi}\left(x-c_{+} t\right)$ is a solution to

$$
u_{t}=\hat{\mu} * u-u+\hat{f}(u) .
$$

So, almost similarly as (4.13), we also obtain

$$
\inf _{\lambda>0} \frac{\int_{y \in \mathbb{R}} e^{\lambda y} d \hat{\mu}(y)-1+\sigma}{\lambda} \leq c_{+} .
$$

Hence, the conclusion of (ii) follows from (4.14).

Lemma 12. Suppose $\mu(\{0\}) \neq 1$. Then,

$$
0<\inf _{\lambda_{-}>0} \frac{\int_{y \in \mathbb{R}}\left(e^{\lambda_{-} y}-1\right) d \mu(y)+\sigma}{\lambda_{-}}+\inf _{\lambda_{+}>0} \frac{\int_{y \in \mathbb{R}}\left(e^{-\lambda_{+} y}-1\right) d \mu(y)+\sigma}{\lambda_{+}}
$$

holds for all $\sigma \in(0,+\infty)$.

Proof. Because $\inf _{\lambda_{-}>0} \int_{y \in \mathbb{R}} e^{\lambda_{-} y} d \mu(y) \neq+\infty$ implies $\lim _{\lambda_{-} \downarrow+0} \int_{y \in \mathbb{R}} e^{\lambda_{-} y} d \mu(y)=\int_{y \in \mathbb{R}} 1 d \mu(y)=1$, we see $\lim _{\lambda_{-} \downarrow+0} \frac{\int_{y \in \mathbb{R}} e^{\lambda_{-} y} d \mu(y)-1+\sigma}{\lambda_{-}}$ $=+\infty$. Hence, if $\inf _{\lambda_{-}>0} \frac{\int_{y \in \mathbb{R}} e^{\lambda_{-} y} d \mu(y)-1+\sigma}{\lambda_{-}} \neq+\infty$ holds, then there exists a positive constant $\varepsilon$ such that

$$
\inf _{\lambda_{-}>0} \frac{\int_{y \in \mathbb{R}} e^{\lambda_{-} y} d \mu(y)-1+\sigma}{\lambda_{-}}=\inf _{\lambda_{-} \geq \varepsilon} \frac{\int_{y \in \mathbb{R}} e^{\lambda_{-} y} d \mu(y)-1+\sigma}{\lambda_{-}} \geq \inf _{\lambda_{-} \geq \varepsilon} \frac{-1}{\lambda_{-}}=-\frac{1}{\varepsilon}
$$

holds. So, we see $\inf _{\lambda_{-}>0} \frac{\int_{y \in \mathbb{R}} e^{\lambda_{-} y} d \mu(y)-1+\sigma}{\lambda_{-}} \neq-\infty$. Similarly, we also see $\inf _{\lambda_{+}>0} \frac{\int_{y \in \mathbb{R}} e^{-\lambda_{+} y} d \mu(y)-1+\sigma}{\lambda_{+}} \neq-\infty$. Therefore, it is sufficient if we show that the inequality (4.15) holds when

$$
\inf _{\lambda_{-}>0} \frac{\int_{y \in \mathbb{R}} e^{\lambda_{-} y} d \mu(y)-1+\sigma}{\lambda_{-}} \neq+\infty
$$

and

$$
\inf _{\lambda_{+}>0} \frac{\int_{y \in \mathbb{R}} e^{-\lambda_{+} y} d \mu(y)-1+\sigma}{\lambda_{+}} \neq+\infty
$$

hold. Suppose (4.16) and (4.17) hold. Then, $\inf _{\lambda_{-}>0} \int_{y \in \mathbb{R}} e^{\lambda_{-} y} d \mu(y) \neq+\infty$ and $\inf _{\lambda_{+}>0} \int_{y \in \mathbb{R}} e^{-\lambda_{+} y} d \mu(y) \neq+\infty$ hold. Hence, because there exist positive constants $\lambda_{-}$and $\lambda_{+}$such that $\int_{y \in \mathbb{R}}\left(e^{\lambda_{-} y}+e^{-\lambda_{+} y}\right) d \mu(y)<+\infty$ holds, we have 


$$
\int_{y \in \mathbb{R}}|y| d \mu(y)<+\infty .
$$

Because of $\mu(\{0\}) \neq \mu(\mathbb{R})$, we see that $\mu((-\infty, 0)) \neq 0$ or $\mu((0,+\infty)) \neq 0$ holds.

Suppose $\mu((-\infty, 0)) \neq 0$. Then, $\lim _{\lambda_{+} \rightarrow+\infty} \frac{\int_{y \in \mathbb{R}}\left(e^{-\lambda_{+} y}-1\right) d \mu(y)+\sigma}{\lambda_{+}}=+\infty$ holds. Hence, there exists $N \in \mathbb{N}$ such that $\inf _{\lambda_{+}>0} \frac{\int_{y \in \mathbb{R}}\left(e^{-\lambda y^{y}}-1\right) d \mu(y)+\sigma}{\lambda_{+}}=$ $\inf _{\lambda_{+} \in(0, N]} \frac{\int_{y \in \mathbb{R}}\left(e^{-\lambda+y}-1\right) d \mu(y)+\sigma}{\lambda_{+}}$holds. So, from (4.18), we have

$$
\begin{aligned}
& \inf _{\lambda_{-}>0} \frac{\int_{y \in \mathbb{R}}\left(e^{\lambda_{-} y}-1\right) d \mu(y)+\sigma}{\lambda_{-}}+\inf _{\lambda_{+}>0} \frac{\int_{y \in \mathbb{R}}\left(e^{-\lambda_{+} y}-1\right) d \mu(y)+\sigma}{\lambda_{+}} \\
& \geq \inf _{\lambda_{-}>0}\left(\int_{y \in \mathbb{R}} y d \mu(y)+\frac{\sigma}{\lambda_{-}}\right)+\inf _{\lambda_{+} \in(0, N]}\left(-\int_{y \in \mathbb{R}} y d \mu(y)+\frac{\sigma}{\lambda_{+}}\right)=\frac{\sigma}{N} .
\end{aligned}
$$

When $\mu((0,+\infty)) \neq 0$, we also have the inequality (4.15) almost similarly as $\mu((-\infty, 0)) \neq 0$.

The following gives sub and super-solutions:

Lemma 13. Let a Lipschitz continuous function $\hat{f}$ on $\mathbb{R}$ satisfy

$$
\hat{f}>0 \quad(u<0), \quad \hat{f}=f \quad(0 \leq u \leq 1), \quad \hat{f}<0 \quad(1<u) .
$$

Let a function $\rho \in C^{1}(\mathbb{R})$ satisfy $\rho=0$ in $(-\infty, 0], \rho=1$ in $[1,+\infty)$ and $\rho^{\prime}>0$ in $(0,1)$. Suppose a positive constant $\varepsilon$ is sufficiently small. Then, the function $\underline{u}(t, x):=\rho\left(\varepsilon x-\frac{t}{\varepsilon}\right)-\frac{1-\alpha}{4}$ is a sub-solution to the equation

$$
u_{t}=\mu * u-u+\hat{f}(u)
$$

and the function $\bar{u}(t, x):=\rho\left(\varepsilon x+\frac{t}{\varepsilon}+1\right)+\frac{\alpha}{4}$ is a super-solution to (4.20).

Proof. We put a positive constant $\delta$ as

$$
\delta:=\min \left\{\min _{u \in\left[-\frac{1-\alpha}{4},-\frac{1-\alpha}{8}\right] \cup\left[1-\frac{1-\alpha}{2}, 1-\frac{1-\alpha}{4}\right]} \hat{f}(u), \min _{u \in\left[\frac{\alpha}{4}, \frac{\alpha}{2}\right] \cup\left[1+\frac{\alpha}{8}, 1+\frac{\alpha}{4}\right]}(-\hat{f}(u))\right\} .
$$

Then, there exists $N \in \mathbb{N}$ such that

$$
\mu(\mathbb{R} \backslash(-N,+N))<\delta
$$


holds. We also put a positive constant $C$ as

$$
C:=\min \left\{\rho^{\prime}(z) \mid \min \left\{\frac{1-\alpha}{8}, \frac{\alpha}{4}\right\} \leq \rho(z) \leq 1-\min \left\{\frac{1-\alpha}{4}, \frac{\alpha}{8}\right\}\right\} .
$$

Suppose a positive constant $\varepsilon$ is small. Then, because

$$
\begin{aligned}
& \left|\int_{y \in \mathbb{R}} \rho(\varepsilon(x-y)+s) d \mu(y)-\rho(\varepsilon x+s)\right| \\
& \quad \leq \int_{y \in \mathbb{R}}|\rho(\varepsilon(x-y)+s)-\rho(\varepsilon x+s)| d \mu(y) \\
& \quad \leq \int_{y \in(-N,+N)}|\rho(\varepsilon(x-y)+s)-\rho(\varepsilon x+s)| d \mu(y)+\mu(\mathbb{R} \backslash(-N,+N)) \\
& \quad \leq \sup _{h \in(-\varepsilon N,+\varepsilon N), z \in \mathbb{R}}|\rho(z+h)-\rho(z)|+\mu(\mathbb{R} \backslash(-N,+N)) \leq \delta
\end{aligned}
$$

holds for all $s$ and $x \in \mathbb{R}$, we have

$$
-(\mu * \underline{u}-\underline{u}) \leq \delta
$$

and

$$
\mu * \bar{u}-\bar{u} \leq \delta
$$

for all $t$ and $x \in \mathbb{R}$. We see that $\rho(z)-\frac{1-\alpha}{4} \in\left[-\frac{1-\alpha}{8}, 1-\frac{1-\alpha}{2}\right]$ implies $\frac{1-\alpha}{8} \leq$ $\rho(z) \leq 1-\frac{1-\alpha}{4}$ and $\rho^{\prime}(z) \geq C$. We also see that $\rho(z)-\frac{1-\alpha}{4} \notin\left[-\frac{1-\alpha}{8}, 1-\frac{1-\alpha}{2}\right]$ implies $\rho(z)-\frac{1-\alpha}{4} \in\left[-\frac{1-\alpha}{4},-\frac{1-\alpha}{8}\right] \cup\left[1-\frac{1-\alpha}{2}, 1-\frac{1-\alpha}{4}\right]$ and $\hat{f}\left(\rho(z)-\frac{1-\alpha}{4}\right) \geq \delta$. Therefore, we have

$$
\frac{1}{\varepsilon} \rho^{\prime}(z)+\hat{f}\left(\rho(z)-\frac{1-\alpha}{4}\right) \geq \min \left\{\frac{1}{\varepsilon} C+\min _{u \in\left[-\frac{1-\alpha}{8}, 1-\frac{1-\alpha}{2}\right]} \hat{f}(u), \delta\right\}=\delta
$$

for all $z \in \mathbb{R}$, as the positive constant $\varepsilon$ is small. So,

$$
-\underline{u}_{t}+\hat{f}(\underline{u}) \geq \delta
$$

holds for all $t$ and $x \in \mathbb{R}$. Similarly, because we also have

$$
\frac{1}{\varepsilon} \rho^{\prime}(z)-\hat{f}\left(\rho(z)+\frac{\alpha}{4}\right) \geq \min \left\{\frac{1}{\varepsilon} C+\min _{u \in\left[\frac{\alpha}{2}, 1+\frac{\alpha}{8}\right]}(-\hat{f}(u)), \delta\right\}=\delta
$$

for all $z \in \mathbb{R}$,

$$
\bar{u}_{t}-\hat{f}(\bar{u}) \geq \delta
$$


holds for all $t$ and $x \in \mathbb{R}$. From (4.21), (4.22), (4.23) and (4.24), we see

$$
-(\mu * \underline{u}-\underline{u}) \leq-\underline{u}_{t}+\hat{f}(\underline{u})
$$

and

$$
\mu * \bar{u}-\bar{u} \leq \bar{u}_{t}-\hat{f}(\bar{u})
$$

for all $t$ and $x \in \mathbb{R}$.

Proof of Theorem 1.

When $\mu(\{0\})=1$, the conclusion of Theorem 1 is trivial. Suppose $\mu(\{0\}) \neq$ 1. Then, in virtue of Lemmas 11,12 and $f^{\prime}(\alpha)>0$, the semiflow $\left\{Q^{t}\right\}_{t \in[0,+\infty)}$ on $\mathcal{M}$ generated by (1.1) satisfies Hypothesis 7 . So, Theorem 8 can work.

We take a Lipschitz continuous function $\hat{f}$ on $\mathbb{R}$ with (4.19). Then, by Lemma 13 , there exist two constants $\underline{c}, \bar{c}$, two bounded, continuous and monotone functions $\hat{\psi}$ and $\hat{\bar{\psi}}$ on $\mathbb{R}$ with $\underline{\hat{\psi}}(0) \in(-\infty, 0), \underline{\hat{\psi}}(+\infty) \in(\alpha, 1), \hat{\bar{\psi}}(-\infty) \in$ $(0, \alpha)$ and $\hat{\bar{\psi}}(0) \in(1,+\infty)$ such that $\underline{u}(t, x):=\underline{\hat{\psi}}(x+\underline{c} t)$ is a sub-solution to (4.20) and $\bar{u}(t, x):=\hat{\bar{\psi}}(x+\bar{c} t)$ is a super-solution to (4.20).

We put $\psi:=\max \{\hat{\psi}, 0\} \in \mathcal{M}$ and $\bar{\psi}:=\min \{\hat{\bar{\psi}}, 1\} \in \mathcal{M}$. Then, $\psi(0)=0$, $\underline{\psi}(+\infty) \in(\alpha, \overline{1}), \bar{\psi}(-\infty) \in(0, \alpha)$ and $\bar{\psi}(0)=1$ hold. Further, $\left(Q^{t}[\underline{\psi}]\right)(x)$ and $\left(Q^{t}[\bar{\psi}]\right)(x)$ are solutions to not only (1.1) but also (4.20) in $t \in[0,+\infty)$. Hence, because $\underline{\hat{\psi}} \leq \underline{\psi}$ and $\bar{\psi} \leq \hat{\bar{\psi}}$ hold, we have

$$
\underline{\psi}(x+\underline{c} t)=\max \{\underline{\hat{\psi}}(x+\underline{c} t), 0\} \leq\left(Q^{t}[\underline{\psi}]\right)(x)
$$

and

$$
\left(Q^{t}[\bar{\psi}]\right)(x) \leq \min \{\hat{\bar{\psi}}(x+\bar{c} t), 1\}=\bar{\psi}(x+\bar{c} t)
$$

for all $t \in[0,+\infty)$. Therefore, by Theorem 8 , there exist $c \in[\underline{c}, \bar{c}]$ and $\phi \in \mathcal{M}$ with $\phi(-\infty)=0$ and $\phi(+\infty)=1$ such that $\left(Q^{t}[\phi]\right)(x-c t) \equiv \phi(x)$ holds for all $t \in[0,+\infty)$. So, $u(t, x):=\phi(x+c t)$ is a solution to (1.1).

\section{Acknowledgments}

I am grateful to an anonymous referee for valuable comments. It was partially supported by Grant-in-Aid for Scientific Research (No. 19740092) from Ministry of Education, Culture, Sports, Science and Technology, Japan. 


\section{References}

[1] N. D. Alikakos, P. W. Bates and X. Chen, Periodic traveling waves and locating oscillating patterns in multidimensional domains, Trans. Amer. Math. Soc. 351 (1999), no. 7, 2777-2805

[2] P. Bates and F. Chen, Periodic traveling waves for a nonlocal integro-differential model, Electron. J. Differential Equations 1999, No. 26, 19 pp. (electronic).

[3] P. W. Bates, X. Chen and A. J. J. Chmaj, Traveling waves of bistable dynamics on a lattice, SIAM J. Math. Anal. 35 (2003), no. 2, 520-546 (electronic).

[4] P. W. Bates and A. Chmaj, A discrete convolution model for phase transitions, Arch. Ration. Mech. Anal. 150 (1999), no. 4, 281-305.

[5] P. W. Bates, P. C. Fife, X. Ren and X. Wang, Traveling waves in a convolution model for phase transitions, Arch. Rational Mech. Anal. 138 (1997), no. 2, 105-136.

[6] X. Chen, Existence, uniqueness, and asymptotic stability of traveling waves in nonlocal evolution equations, Adv. Differential Equations 2 (1997), no. 1, 125-160.

[7] X. Chen, J.-S. Guo and C.-C. Wu, Traveling waves in discrete periodic media for bistable dynamics, Arch. Ration. Mech. Anal. 189 (2008), no. 2, 189-236.

[8] A. J. J. Chmaj, Existence of traveling waves for the nonlocal Burgers equation, Appl. Math. Lett. 20 (2007), no. 4, 439-444.

[9] S.-N. Chow, J. Mallet-Paret and W. Shen, Traveling waves in lattice dynamical systems, J. Differential Equations 149 (1998), no. 2, 248-291.

[10] R. Coutinho and B. Fernandez, Fronts in extended systems of bistable maps coupled via convolutions, Nonlinearity 17 (2004), no. 1, 23-47.

[11] J. Coville, On uniqueness and monotonicity of solutions of non-local reaction diffusion equation, Ann. Mat. Pura Appl. (4) 185 (2006), no. 3, 461-485.

[12] $\ldots$, Travelling fronts in asymmetric nonlocal reaction diffusion equation: The bistable and ignition case, preprint.

[13] P. C. Fife and J. B. McLeod, A phase plane discussion of convergence to travelling fronts for nonlinear diffusion, Arch. Rational Mech. Anal. 75 (1980/81), no. 4, 281-314.

[14] F. Hamel and S. Omrani, Existence of multidimensional travelling fronts with a multistable nonlinearity, Adv. Differential Equations 5 (2000), no. 4-6, 557-582.

[15] J. Mallet-Paret, The global structure of traveling waves in spatially discrete dynamical systems, J. Dynam. Differential Equations 11 (1999), no. 1, 49-127.

[16] A. Volpert and V. Volpert, Existence of multidimensional travelling waves and systems of waves, Comm. Partial Differential Equations 26 (2001), no. 3-4, 421-459.

[17] H. F. Weinberger, Long-time behavior of a class of biological models, SIAM J. Math. Anal. 13 (1982), no. 3, 353-396.

[18] H. Yagisita, Existence and nonexistence of traveling waves for a nonlocal monostable equation, Publ. Res. Inst. Math. Sci., submitted. 